\title{
Molecular Architecture for Autonomic Wireless Mesh Networks
}

\author{
Mohammad Nassiri, Fabrice Theoleyre, Martin Heusse, and Andrzej Duda \\ LIG - Grenoble Informatics Laboratory \\ Grenoble, France \\ \{nassiri, theoleyr, heusse, duda\}@imag.fr
}

\section{INTRODUCTION}

We consider wireless mesh networks that use 802.11 wireless LANs for interconnecting mesh routers. When mesh routers use only one network interface and a single radio channel, performance of packet forwarding quickly degrades with the number of hops due to channel contention and spatial problems such as hidden, exposed, masked, and blocked nodes. We observe that capacity of a wireless mesh network strongly depends on the ability of nearby mesh routers to communicate in parallel, which is only possible if the neighbor routers that may interfere use different channels.

The goal of this paper is to propose a mesh architecture for autonomic assignment and use of multiple channels to improve network capacity. We seek an autonomous and distributed way of operation-mesh routers organize themselves to form a desired mesh topology and take specific roles to achieve high performance. The architecture is coupled with an efficient mechanism for packet forwarding over multiple wireless links that takes advantage of such a mesh network. We adopt a molecular analogy for defining our architecture.

\section{MOLECULAR ARCHITECTURE}

Our model assumes that each mesh router has only one wireless network interface that can switch channels. However we can easily generalize it to support mesh routers with multiple interfaces.

We adopt a molecular analogy for defining our architecture (cf. Figure 1). An atom is a basic entity from which we construct a mesh network. It is composed of a nucleus, a mesh router that uses a fixed channel for communicating with electrons, its immediate neighbors.

Permission to make digital or hard copies of all or part of this work for personal or classroom use is granted without fee provided that copies are not made or distributed for profit or commercial advantage and that copies bear this notice and the full citation on the first page. To copy otherwise, to republish, to post on servers or to redistribute to lists, requires prior specific permission and/or a fee.

CoNEXT'07, December 10-13, 2007, New York, NY, U.S.A

Copyright 2007 ACM 978-1-59593-770-4/ 07/ 0012 ...\$5.00.

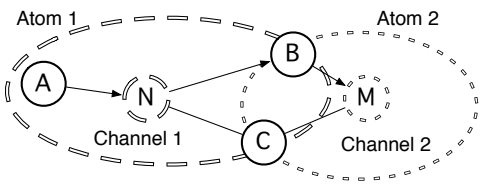

Figure 1: Two atoms sharing two electrons

Atoms bond together to form a molecule, which corresponds to a connected wireless mesh network. Electrons belong to adjacent atoms and communicate with their nuclei. There is no direct link between two electrons nor between two nuclei, e.g. mesh routers $\mathrm{N}$ and $\mathrm{M}$ in Figure 1 are nuclei of two atoms bonded by two mesh routers corresponding to electrons B and C.

There is a possible contention in an atom between entities that use the same channel (e.g. N, A, B, and C). Mesh routers can use any suitable MAC access method to control contention in an atom such as the standard IEEE DCF or any of its proposed optimizations.

To interconnect atoms and achieve high capacity, electrons switch between channels used by neighbor nuclei so that packets going through adjacent atoms benefit from parallel communications. During the construction of the mesh, a nucleus dynamically chooses its channel depending on the channels used in its neighborhood by other atoms. By choosing different channels, two neighbor atoms that may interfere when using the same channel limit interference problems, which contributes to achieving high capacity.

When neighbor nodes are tuned to possibly different channels, the problem of deafness arises: a node is trying to send its frame on one channel and its receiver is listening to another one. In our architecture, a nucleus node chooses its channel and lets it know to all electrons in its vicinity. The nucleus always stays on this channel: no deafness is possible. We define a molecular frame forwarding mechanism for communication between a nucleus and an electron that takes advantage of different channels used in adjacent atoms: an electron sends a CTS control frame to pull a frame - it tells a nucleus that it is ready to receive a frame on the nucleus 
channel. As it is up to an electron to pull a frame, an electron cannot be deaf, but the nucleus needs to notify its electrons that there are pending frames for them. To achieve this, the nucleus piggybacks the information on the destinations of pending frames onto data, ACK, and beacon frames.

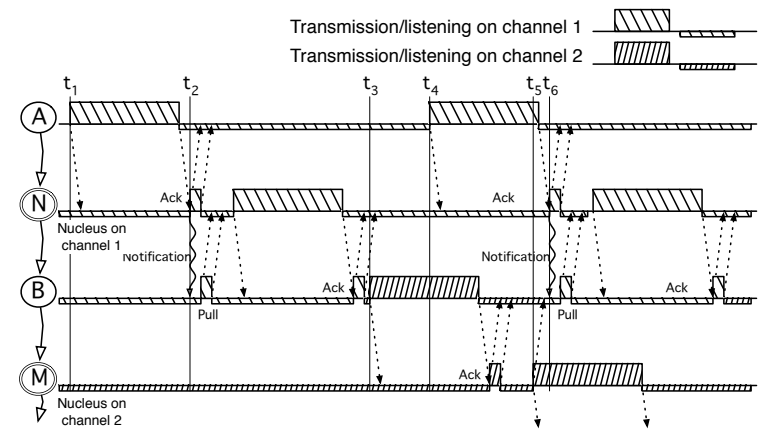

Figure 2: Frame exchanges during packet forwarding in a chain.

One important issue is to construct the mesh molecules. We want a mesh molecule composed of interconnected atoms with one nucleus per atom. Interconnection of adjacent atoms relies on one or more electrons common to two adjacent atoms with nuclei 2 hops apart. The molecular mesh structure may lead to the elimination of some links (e.g. to avoid a direct link between two electrons or two nuclei). At the same time, we want the resulting mesh molecule to remain connected. This kind of structure corresponds to the Independent Sets and more precisely to the Weakly Connected Dominating Set (WCDS) [3]. We construct our mesh molecule in a distributed and localized manner.

The proper operation of a mesh molecule requires the knowledge of neighbor nodes: their state (nucleus or electron) and the channel to use for communication. As neighbor nodes may belong to different atoms and use different channels to transmit data, we define a multichannel neighbor discovery protocol.

\subsection{Frame Forwarding Mechanism}

Figure 2 illustrates the behavior of our mechanism for frame forwarding. When A (an electron) has a frame to send to $\mathrm{N}$ (a nucleus), it accesses the channel conforming to the channel access method and transmits the frame on the channel used by the nucleus. The transmission does not suffer from deafness, because the nucleus always listens to the chosen channel. By piggybacking the information on the frame destinations present in its buffer (B, another electron) onto the data and ACK frames, $\mathrm{N}$ notifies $\mathrm{B}$ at instant $t_{2}$ that there is a pending frame to receive. Thus, B stays on the channel used by $\mathrm{N}$, requests the frame from $\mathrm{N}$, and receives it. Then, at instant $t_{3}$, B forwards the packet contained in the frame to $M$ using the channel chosen by $M$ (channel 2).
When $\mathrm{M}$ is further forwarding the packet on channel 2 , B finds out that it is not involved in this transmission: it may change channels at instant $t_{5}$ to receive notifications from its other nucleus at instant $t_{6}$.

\section{PRELIMINARY RESULTS}

We provide some preliminary results on performance of our molecular mesh network. We can compare packet

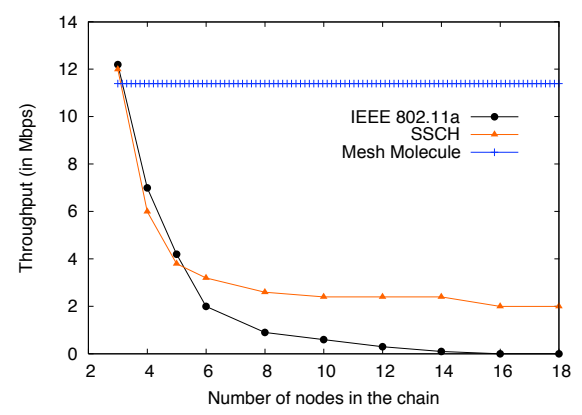

Figure 3: Throughput for different forwarding schemes.

forwarding performance over a chain topology, which is a first test case for mesh networks. Figure 3 compares the expected performance of 802.11a DCF in a traditional multi-hop ad hoc wireless networks [2] with SSCH [1] and our proposal. The throughput values for 802.11a and SSCH are extracted from the SSCH proposal [1] that considers a chain of several nodes. Our results come from the following observation. In the chain configuration, packets go through several atoms interconnected by an electron. Packet forwarding benefits from parallel communication in adjacent atoms: in one atom, the nuclei and the electron share the same channel so the resulting throughput will be the half of the throughput of a single hop. In different atoms, frames use different channels so they are forwarded in parallel. There is also a slight overhead related to the use of the CTS control frame.

\section{REFERENCES}

[1] V. Bahl, R. Chandra, and J. Dunagan. SSCH: Slotted Seeded Channel Hopping For Capacity Improvement in IEEE 802.11 Ad Hoc Wireless Networks. In Proc. MOBICOM, pages 216-230, Philadelphia, USA, October 2004. ACM.

[2] C. Chaudet, D. Dhoutaut, and I. Guerin-Lassous. Performance Issues with IEEE 802.11 in Ad Hoc Networking. IEEE Communications Magazine, 43(7):110-116, July 2005.

[3] J. E. Dunbar, J. W. Grossman, J. H. Hattingh, S. T. Hedetniemi, and A. A. McRae. On Weakly Connected Domination in Graphs. Discrete Mathematics, 167-168:261-269, April 1997. 